\title{
Presencia física de profesionales de Salud Mental en un Centro de Atención Primaria como forma alternativa de coordinación. Una experiencia piloto.
}

\section{Physical presence of mental health professionals in a pri- mary care centre as an alternative way of coordination. A pilot experience.}

\section{RESUMEN:}

Introducción: Problemas de coordinación entre los niveles de atención primaria y salud mental sugieren la necesidad de explorar nuevas vías que faciliten la comunicación entre ambos niveles asistenciales.

Material y Método: Un Psiquiatra y un Psicólogo Clínico de Alcalá de Henares (Área 3 de la Comunidad Autónoma de Madrid) se han desplazado un día a la semana a un centro de atención primaria (centro experimental) para evaluar pacientes derivados por los médicos de ese centro y contrastar directamente con ellos sus valoraciones. Después de 15 meses de funcionamiento, se administró a los médicos un cuestionario acerca de su satisfacción con su relación con salud mental. Sus respuestas se compararon con las de médicos de otro centro en el que no tenía lugar la experiencia (centro control).

Resultados: los médicos del centro experimental valoraban mejor la información recibida por salud mental y la cantidad de contactos que tenían con ellos, percibían al equipo de salud mental como más disponible, recibían más cursos específicos, se habían coordinado más con salud mental y, en general, valoraban mejor la coordinación atención primaria - salud mental que los del centro control.

Conclusiones: La experiencia muestra cómo es posible una forma alternativa de coordinación con la que los médicos de primaria se muestran altamente satisfechos. Se plantean algunas cuestiones metodológicas y posibles vías para continuar futuros trabajos.

PALABRAS CLAVE: Atención Primaria de Salud; Salud Mental; Coordinación

\section{ABSTRACT: \\ Introduction: Coordination problems bet-} ween primary care and mental health levels suggest the need to explore new ways to improve communication between both levels.

Procedures: A psychiatrist and a clinical psychologist in Alcalá de Henares, Madrid, moved to a primary care centre (experimental centre) once a week in order to assess patients referred by the general practitioners there and share afterwards their views with them. After 15 months working this way, general practitioners were given a survey about their satisfaction with their relation to mental health. Their answers were compared with those of the staff from another centre (control centre).

Outcome: the staff in the experimental centre valued more positively than the staff in the control centre the following aspects: quality of information received from mental health, amount of contacts with mental health, availability of mental health staff, specific formation on mental health and coordination with mental health staff.

Discussion: This experience shows how it is possible to develop an alternative way of coordination between mental health and primary care with which general practitioners feel highly satisfied. Methodological issues as well as possible ways for future works are discussed.

KEYWORDS: Primary Health Care; Mental Health; Coordination. 


\section{Introducción.}

En los últimos años del siglo XX se produjo en nuestro país una profunda transformación del sistema de atención a la salud mental, que siguió la estela de lo ocurrido desde los años sesenta en otros países desarrollados ${ }^{1}$, y que, en líneas generales se ajustó al modelo propuesto en el Documento de la Comisión Ministerial para la Reforma Psiquiátrica del año $1985^{2}$ y que se plasmó en el artículo 20 de la Ley General de Sanidad ${ }^{3}$.

La reforma psiquiátrica supuso la transición desde un sistema de atención que sólo ofrecía, para los trastornos mentales graves, internamiento en los hospitales monográficos de las diputaciones provinciales, al margen del sistema sanitario general, y que prácticamente desatendía los trastornos llamados menores, a uno que pretendía proveer atención en la comunidad desde una red compleja de dispositivos alternativos a la hospitalización e integrados en el sistema sanitario general $^{4}$. La atención en Salud Mental pasó a ser principalmente ambulatoria y el paciente psiquiátrico, hasta entonces un paria, fue equiparado, al menos sobre el papel, al resto de pacientes atendidos por otras condiciones médicas.

El fin último del nuevo modelo sería proporcionar al paciente psiquiátrico una atención personalizada e integral en su entorno, garantizando la continuidad de los cuidados y reduciendo al máximo posible la necesidad de hospitalización. Ésta, cuando tuviera que hacerse, sería preferentemente de carácter breve y tendría lugar en las unidades psiquiátricas de los hospitales generales. Además del centro de salud mental, piedra angular del nuevo sistema, se desarrollan toda una serie de recursos dirigidos a la rehabilitación y reinserción del paciente psiquiátrico en la comunidad ${ }^{2,4}$.

Con el tiempo, conforme se van detectando nuevas necesidades, aparecen nuevos dispositivos (hospitales de día, recursos residenciales de distintos tipos, programas de cuidados comunitarios para pacientes graves...), y éstos tienden a ser más específicos. Este desarrollo de la red, no obstante, implica una mayor necesidad de coordinación entre los diferentes recursos y, además, visto en perspectiva, hace preguntarse hasta qué punto no se están remediando problemas a base de llenar el sistema de parches, en vez de plantearse cambios estructurales que conduzcan a una atención comunitaria más eficaz y eficiente.

Sin duda la Reforma Psiquiátrica ha traído muchos resultados positivos, siendo el principal que el paciente psiquiátrico grave puede disfrutar en la actualidad de una atención y una calidad de vida impensables hace sólo veinticinco años. Por otro lado, no todo el monte es orégano; cabos sueltos y faltas de previsión procedentes de los comienzos han conducido, desde los inicios, a problemas ante los que es necesario plantear nuevas soluciones ${ }^{5}$. La Estrategia en Salud Mental del Sistema Nacional de Salud, aprobada en 2007, reconoce esta situa- 
ORIGINALES Y REVISIONES

ción y propone unas líneas maestras para buscarlas ${ }^{6}$.

La reforma respondió mejor a las necesidades del paciente psiquiátrico considerado "grave" (es decir, diagnosticado de trastorno psicótico), y sus promotores trabajaron sobre la idea de que el resto de "trastornos mentales menores" (principalmente cuadros ansiosos y depresivos, sin perjuicio de la gravedad que para los pacientes así diagnosticados puedan tener esas condiciones) serían manejados por la atención primaria. Muy desde el inicio pudo comprobarse, sin embargo, que los profesionales de atención primaria no contaban con la formación, tiempo ni disposición necesarios para atender adecuadamente a estos pacientes ${ }^{5}$. Los términos en que se produciría la coordinación entre atención primaria y salud mental no quedaron explícitamente regulados, por lo que cuando esta coordinación se daba, quedaba supeditada al buen entendimiento y disponibilidad de profesionales concretos en ambos niveles. El papel de la atención primaria en la atención a la salud mental y su relación con los recursos de atención especializada ha debido constituirse, de hecho, en uno de los principales problemas a abordar por la Estrategia en Salud Mental de $2007^{6}$.

Los profesionales de atención especializada se encuentran saturados de pacientes que, sin perjuicio de lo genuino de su malestar, son considerados "light" y, por tanto, asumibles por atención primaria; se crea entre los profesionales de salud mental una imagen del médico de atención primaria como "poco contenedor", que "se angustia enseguida en cuanto el paciente le llora" y se cuestiona la artificiosidad de separar lo psicológico de lo médico/orgánico.

Por otro lado los médicos de atención primaria se encuentran igualmente con un volumen de demanda difícil de manejar: parece que todas las especialidades estén empeñadas en que sean ellos los que lleven los casos "menores". La problemática emocional del paciente puede resultar incómoda y, sobre todo, consume tiempo de consulta (con los consiguientes retrasos y cabreos de los pacientes que están en la sala de espera). Ante este tipo de pacientes al médico de atención primaria pueden surgirle numerosas dudas que no tiene con quién consultar, al menos de forma inmediata. En numerosos casos los criterios de derivación a la atención especializada están muy poco claros. Y, además, la respuesta recibida a veces por parte de los profesionales de salud mental denota que estos consideran que la derivación no ha sido efectuada adecuadamente. Es frecuente que un paciente remitido a salud mental sea devuelto a atención primaria tras una única consulta, y las indicaciones del profesional de salud mental no siempre le son de ayuda al MAP para continuar tratando la problemática del paciente en su consulta.

Entre uno y otro nivel asistencial se encuentran los pacientes que, van y vienen con la sensación de que nadie se hace cargo de su situación. Mientras tanto, la lista de espera para derivación a atención especializada se alarga, como lo hace 
también el intervalo de tiempo entre sesiones una vez que al paciente se le hace un seguimiento en salud mental.

Si ante esta situación hubiera que elegir un foco terapéutico de trabajo desde el esquema de la Terapia Interpersonal para la Depresión (TIP) ${ }^{7}$, habría que decir que, sin duda, se trata de un caso claro de Disputa Interpersonal (relación significativa en la que las expectativas de cada integrante sobre la misma no son recíprocas) que actualmente se encuentra en estado de impasse (punto muerto en el que no hay comunicación entre las partes y el malestar queda latente). De acuerdo con la TIP, en este punto sería importante reabrir los canales de comunicación y permitir que la disputa latente se haga franca, de manera que cada una de las partes pueda ajustar sus expectativas respecto del otro.

Actualmente la principal herramienta de comunicación entre ambos niveles es el Parte de InterConsulta (PIC), en el que el médico de atención primaria describe, a veces muy escuetamente, el caso y el motivo de la derivación, y que el propio paciente lleva en su primera cita al profesional de salud mental. Éste, finalizada la evaluación del paciente, contesta a la instancia derivadora en el mismo PIC, a veces de manera igual de escueta, y de nuevo es el paciente el encargado de hacerlo llegar de vuelta a su médico de cabecera. Caso de continuar el paciente en seguimiento en salud mental, pocas veces vuelven a tener contacto los profesionales de ambos niveles de cara a un trabajo conjunto sobre ese caso. Lo poco fluido de esta comunicación puede ser un factor importante a la hora de entender por qué los niveles de atención primaria y salud mental acaban funcionando muchas veces en paralelo y centrados cada uno en sí mismos.

Como alternativa a este funcionamiento se ha planteado a veces una forma de coordinación alternativa entre ambos niveles asistenciales ${ }^{8,9}$. Siguiendo esta línea, en los Servicios de Salud Mental del Área 3 de Madrid se realizó una experiencia piloto consistente en trasladar la evaluación de los nuevos pacientes por el profesional de salud mental al mismo centro de atención primaria desde el que se producía la derivación. Esto daría la oportunidad de que el especialista de salud mental y el médico de atención primaria pudieran contrastar sus visiones sobre el caso en un encuentro personal, quizás el mismo día que el especialista hubiera evaluado al paciente en cuestión. Asimismo, el especialista de salud mental estaría en una posición inmejorable para detectar aquellos aspectos de la labor asistencial del médico de atención primaria que pudieran beneficiarse de seminarios de formación en salud mental (manejo de la entrevista clínica, formación en psicoterapia de apoyo, manejo de psicofármacos, dudas diagnósticas, etc.). El objetivo principal de la experiencia era comprobar la viabilidad de un modelo alternativo de relación entre la atención especializada en salud mental y la atención primaria, y recoger, igualmente, información preliminar sobre el impacto en los médicos de atención primaria que participaron. Se presentan asimismo datos 
ORIGINALES Y REVISIONES

preliminares sobre el impacto de esta experiencia en la tasa de derivaciones efectuadas por el centro de atención primaria donde se llevó a cabo la experiencia.

\section{Material y método.}

\subsection{Intervención}

Un psiquiatra y un psicólogo clínico del equipo de salud mental de adultos de Alcalá de Henares se desplazaron durante un día a la semana al Centro de Salud María de Guzmán (la experiencia comenzó en Agosto de 2006 y continuó sin cambios hasta Septiembre de 2008, momento en que se comenzó a ampliar la experiencia a otros centros). En este día evaluaban un máximo de 5 pacientes nuevos adscritos a ese centro de salud (frente al máximo de 8 pacientes nuevos semanales que evaluaban en el centro de salud mental antes de comenzar la experiencia); los profesionales de atención primaria tenían ocasión de asistir con el paciente a la evaluación o de comentarlo en una reunión con los profesionales de salud mental al final de la jornada, donde también podían plantear otras dudas, como la conveniencia de nuevas derivaciones, la forma de realizarlas, posibilidades de manejo de situaciones difíciles en pacientes derivados o no a salud mental, etc.

Se acordaron, además, dos maneras de gestionar la derivación de casos urgentes: de manera presencial (en la reunión semanal entre Primaria-Salud Mental, preparando la cita en el siguiente día de evaluación), o por medio de consultas telefónicas a los profesionales de salud mental el resto de la semana. Para aquellos casos cuya situación clínica no permitiese demorar la evaluación una semana, se mantenía la habitual derivación al Servicio de Urgencias del Hospital Universitario Príncipe de Asturias.

Posteriormente se planteó la realización periódica de seminarios de formación específica en salud mental, según las necesidades que se fueron detectando. Así, se llevaron a cabo reuniones con una periodicidad trimestral, en las que se abordaron aspectos teóricos en relación a la salud mental a propósito de algún caso práctico, o bien se realizaron supervisiones de pacientes, compartidos o no con el equipo de Salud Mental; estas reuniones sirvieron también para revisar con los profesionales de atención primaria la marcha de este programa de enlace. Asimismo, se realizó un curso orientado al entrenamiento en habilidades de entrevista.

\subsection{Sujetos}

Médicos de Atención Primaria de los Centros de Salud María de Guzmán centro objeto del experimento - $(\mathrm{n}=11)$ y Luis Vives - centro utilizado como control - $(n=10)$. Se incluyeron a los profesionales tanto del turno de mañana como 
del turno de tarde. Se excluyeron enfermeros, médicos residentes y pediatras.

2.3. Instrumentos

El principal instrumento de evaluación fue una encuesta escrita ad hoc diseñada de acuerdo con los contenidos considerados más relevantes de cara a la coordinación entre niveles asistenciales, y también basada en encuestas que se habían realizado en otros trabajos ${ }^{10-12}$ (instrumento incluido en el anexo). La información recogida se agrupa en cinco categorías:

- Descripción de la muestra.

- Características de la problemática de SM y su manejo en AP.

- Valoración del MAP sobre la calidad de la información proveniente de SM.

- Valoración del MAP sobre la coordinación AP-SM.

- Percepción de necesidades formativas en SM.

2.4. Procedimiento

Tras quince meses en funcionamiento, se administró esta encuesta a los médicos del centro. Sus respuestas fueron comparadas con las de otro centro, de características similares, en el que no estaba teniendo lugar la experiencia (Centro de Salud Luis Vives, también perteneciente al municipio de Alcalá de Henares).

2.5. Análisis de datos

Se calcularon las medias y desviaciones típicas de las variables cuantitativas (respuestas de las escalas Likert y puntuaciones de las valoraciones subjetivas). Las respuestas de tipo cualitativo (a las preguntas abiertas) fueron clasificadas y agrupadas por un evaluador en diferentes categorías semánticas, y se calculó la frecuencia de aparición de las distintas categorías. En cada ítem cualitativo, además, se añadió una categoría miscelánea ("Otros") en la que se incluían aquellas respuestas que no habían conseguido una frecuencia mayor que 2 en el conjunto total de la muestra.

Se llevaron a cabo pruebas $\chi 2$ sobre tablas de contingencia para comprobar si existían diferencias entre los dos centros en la frecuencia de distribución de los sujetos por las distintas categorías de respuesta (ver Tabla 1 para un resumen del tratamiento estadístico aplicado a cada variable). El análisis de datos se llevó a cabo con ayuda del programa informático SPSS versión 12.0.

\section{Resultados.}

3.1. Descripción de la muestra

La media de edad en ambos grupos superaba los 40 años; asimismo, los médicos de ambos centros llevaban una media de más de 10 años trabajando en el Sistema Nacional de Salud. Aunque la proporción de género y de personal fijo/ 
interino es distinta en cada centro, estas diferencias no alcanzan la significación estadística manteniendo el criterio $\alpha<0,05$ (ver Tabla 1 ).

Tabla 1

Descripción de la muestra

\begin{tabular}{|c|c|c|c|c|}
\hline & \multicolumn{2}{|c|}{$\operatorname{Media}^{\mathrm{a}}(\mathrm{DT}) / \mathrm{n}^{\mathrm{b}}(\%)$} & \multirow[b]{2}{*}{$\mathrm{U} / \mathrm{x}^{2}$} & \multirow[b]{2}{*}{ sig. ${ }^{\mathrm{c}}$} \\
\hline & $\begin{array}{c}\mathrm{M}^{\mathrm{a}} \text { de Guzmán } \\
(\mathrm{n}=11)\end{array}$ & $\begin{array}{c}\text { Luis Vives } \\
(\mathrm{n}=10)\end{array}$ & & \\
\hline Edad & $46,4(9)$ & $41,7(6,9)$ & $\mathrm{U}=35,5$ & 0,295 \\
\hline $\begin{array}{l}\text { Género } \\
\text { Varón } \\
\text { Mujer }\end{array}$ & $\begin{array}{l}4(36,4 \%) \\
7(63,6 \%)\end{array}$ & $\begin{array}{l}1(11,1 \%) \\
8(88,9 \%)\end{array}$ & $x^{2}=1,68$ & 0,319 \\
\hline $\begin{array}{l}\text { Años como MAP } \\
\text { en Sector Público }\end{array}$ & $16,4(7)$ & $13,9(6,3)$ & $\mathrm{U}=37$ & 0,37 \\
\hline $\begin{array}{l}\text { Situación laboral } \\
\text { Fijo } \\
\text { Interino } \\
\end{array}$ & $\begin{array}{l}6(54,5 \%) \\
5(45,5 \%)\end{array}$ & $\begin{array}{l}7(77,8 \%) \\
2(22,2 \%)\end{array}$ & $\mathrm{x}^{2}=1,17$ & 0,374 \\
\hline $\begin{array}{l}\text { Turno de trabajo } \\
\text { Mañana } \\
\text { Tarde }\end{array}$ & $\begin{array}{l}4(40 \%) \\
6(60 \%)\end{array}$ & $\begin{array}{l}5(50 \%) \\
5(50 \%)\end{array}$ & $x^{2}=0,2$ & 1 \\
\hline
\end{tabular}

a Aunque el estadístico U de Mann-Whitney no se calcula sobre las medias muestrales, se exponen éstas en la tabla para facilitar la comprensión.

b La suma de casos en cada valor puede ser menor que la suma total de casos debido a la presencia de valores perdidos en la muestra.

c Todos los contrastes son bilaterales.

3.2. Características de la problemática de salud mental y su manejo en atención primaria

Los médicos de atención primaria de ambos centros coinciden en señalar los problemas de ansiedad y los de depresión/distimia como aquellas problemáticas de salud mental que con más frecuencia acuden a su consulta (Tabla 2). Asimismo, los médicos de atención primaria del centro experimental detectan una gran cantidad de problemáticas adaptativas, esto es, estados mixtos de carácter ansioso-depresivo en el contex to de situaciones de cambio que exigen al paciente un esfuerzo de ajuste ante la nueva situación (lo que podría llamarse "patología de la vida cotidiana"). 
Tabla 2

Características de la problemática de SM y su manejo en AP

\begin{tabular}{|c|c|c|c|c|}
\hline & \multicolumn{2}{|c|}{ Media $^{a}(\mathrm{DT}) / \mathrm{n}(\%)$} & \multirow[b]{2}{*}{$\mathrm{U} / \mathrm{x}^{2}$} & \multirow[b]{2}{*}{ sig. ${ }^{b}$} \\
\hline & $\begin{array}{c}\mathrm{M}^{\mathrm{a}} \text { de Guzmán } \\
(\mathrm{n}=11)\end{array}$ & $\begin{array}{l}\text { Luis Vives } \\
(\mathrm{n}=10)\end{array}$ & & \\
\hline $\begin{array}{l}\text { Problemáticas de SM más frecuentes en AP. } \\
\text { Ansiedad } \\
\text { Depresión/Distimia } \\
\text { Trastorno adaptativo (incluye duelo) } \\
\text { Somatizaciones } \\
\text { Otros } \\
\end{array}$ & $\begin{array}{l}10(90,9 \%) \\
9(81,8 \%) \\
7(63,6 \%) \\
2(18,2 \%) \\
2(18,2 \%) \\
\end{array}$ & $\begin{array}{c}10(100 \%) \\
10(100 \%) \\
2(20 \%) \\
0 \\
2(20 \%) \\
\end{array}$ & $\begin{array}{l}x^{2}=0,95 \\
x^{2}=2,01 \\
x^{2}=4,07 \\
x^{2}=2,01 \\
x^{2}=0,01\end{array}$ & $\begin{array}{l}0,524 \\
0,262 \\
0,056 \\
0,262 \\
0,669\end{array}$ \\
\hline $\begin{array}{l}\text { Criterios de derivación a Atención Especializada de SM } \\
\text { No respuesta a fármacos } \\
\text { Petición del paciente } \\
\text { Mayor gravedad/cronicidad } \\
\text { Mala evolución } \\
\text { Falta de conocimientos } \\
\text { Mala relación médico-paciente } \\
\text { Trastornos psicóticos } \\
\text { Otros } \\
\end{array}$ & $\begin{array}{l}6(54,5 \%) \\
5(45,5 \%) \\
5(45,5 \%) \\
2(18,2 \%) \\
3(27,3 \%) \\
2(18,2 \%) \\
1(9,1 \%) \\
8(72,7 \%) \\
\end{array}$ & $\begin{array}{l}7(70 \%) \\
5(50 \%) \\
2(20 \%) \\
3(30 \%) \\
1(10 \%) \\
1(10 \%) \\
2(20 \%) \\
3(30 \%)\end{array}$ & $\begin{array}{l}\mathrm{x}^{2}=0,53 \\
\mathrm{x}^{2}=0,043 \\
\mathrm{x}^{2}=1,52 \\
\mathrm{x}^{2}=0,403 \\
\mathrm{x}^{2}=1,014 \\
\mathrm{x}^{2}=0,286 \\
\mathrm{x}^{2}=0,509 \\
\mathrm{x}^{2}=3,834\end{array}$ & $\begin{array}{c}0,392 \\
0,59 \\
0,221 \\
0,45 \\
0,331 \\
0,538 \\
0,462 \\
0,063\end{array}$ \\
\hline $\begin{array}{l}\text { Valoración del MAP de su propio trabajo con } \\
\text { pacientes de SM (0 Muy mala-5 muy satisfactoria) }\end{array}$ & $3,45(0,52)$ & $3,33(0,5)$ & $\mathrm{U}=43,5$ & 0,656 \\
\hline $\begin{array}{l}\text { Manejo del paciente SM atendido en AP } \\
\text { (0 Nunca-4 Siempre) } \\
\text { Tratamiento farmacológico } \\
\text { Apoyo psicológico no estructurado } \\
\text { Abordaje psicológico estructurado } \\
\end{array}$ & $\begin{array}{c}2,64(0,5) \\
2,82(1,17) \\
0,64(1,03)\end{array}$ & $\begin{array}{c}3(0) \\
2,4(1,43) \\
0(0)\end{array}$ & $\begin{array}{l}\mathrm{U}=35 \\
\mathrm{U}=46 \\
\mathrm{U}=35\end{array}$ & $\begin{array}{l}0,173 \\
0,557 \\
0,173 \\
\end{array}$ \\
\hline $\begin{array}{l}\text { Percepción del tipo de ayuda que el MAP necesitaría } \\
\text { de SM para el manejo de estos pacientes en AP } \\
\text { Formación en manejo psicoterapéutico } \\
\text { Consulta de dudas sobre pacientes propios } \\
\text { Mejora de la entrevista clínica } \\
\text { Otros }\end{array}$ & $\begin{array}{l}5(45,5 \%) \\
3(27,3 \%) \\
2(18,2 \%) \\
6(54,5 \%)\end{array}$ & $\begin{array}{c}1(10 \%) \\
0 \\
1(10 \%) \\
4(40 \%)\end{array}$ & $\begin{array}{l}x^{2}=3,226 \\
x^{2}=3,182 \\
x^{2}=0,286 \\
x^{2}=0,444\end{array}$ & $\begin{array}{l}0,094 \\
0,124 \\
0,538 \\
0,410\end{array}$ \\
\hline $\begin{array}{l}\text { Percepción subjetiva del MAP de los pacientes } \\
\text { con problemática de SM. } \\
\text { Falta de tiempo } \\
\text { Reacción positiva de interés } \\
\text { Capacidad positiva de manejo } \\
\text { Incomodidad } \\
\text { Capacidad negativa de manejo } \\
\text { Otros }\end{array}$ & $\begin{array}{l}4(36,4 \%) \\
4(36,4 \%) \\
3(27,3 \%) \\
1(9,1 \%) \\
2(18,2 \%) \\
4(36,4 \%)\end{array}$ & $\begin{array}{l}3(30 \%) \\
2(20 \%) \\
3(30 \%) \\
2(20 \%) \\
1(10 \%) \\
3(30 \%)\end{array}$ & $\begin{array}{l}\mathrm{x}^{2}=0,095 \\
\mathrm{x}^{2}=0,687 \\
\mathrm{x}^{2}=0,019 \\
\mathrm{x}^{2}=0,509 \\
\mathrm{x}^{2}=0,286 \\
\mathrm{x}^{2}=0,095\end{array}$ & $\begin{array}{l}0,562 \\
0,367 \\
0,633 \\
0,462 \\
0,538 \\
0,562\end{array}$ \\
\hline
\end{tabular}

a Aunque el estadístico U de Mann-Whitney no se calcula sobre las medias muestrales, se exponen éstas en la tabla para facilitar la comprensión.

b Todos los contrastes son bilaterales. 
La falta de respuesta al tratamiento farmacológico es el principal criterio del médico para decidir derivar un paciente con problemática de salud mental al nivel de atención especializada. Asimismo, cerca de la mitad de derivaciones de ambos centros se realizan a petición del propio paciente, y cerca de la mitad en el centro experimental tenía que ver con la mayor gravedad o cronicidad del caso.

Los médicos de ambos centros valoran aproximadamente por igual su actuación con este tipo de pacientes (puntúan en lugares intermedios de la escala), y a la hora de manejar en su consulta problemática de salud mental recurren tanto a psicofármacos como al apoyo psicológico no estructurado. El uso de algún abordaje psicológico estructurado es mínimo.

Curiosamente casi un $50 \%$ de los médicos del centro experimental consideran que necesitarían mayor formación en este sentido (frente a sólo el 10\% del centro control que echa de menos este tipo de formación). En general, es en el centro experimental donde tiene lugar una mayor demanda de ayuda por parte del equipo de salud mental, pese a que es en ese centro donde se está realizando la experiencia de colaboración atención primaria - salud mental. Más adelante se ofrece una posible explicación de esta aparente paradoja. En cualquier caso, ninguna de estas diferencias resulta estadísticamente significativa manteniendo el criterio $\alpha<0,05$.

Las reacciones de los médicos de atención primaria de ambos centros frente a pacientes de salud mental son variadas y de signo contrapuesto: frente a respuestas que denotan interés (36,4\% en el centro experimental y $20 \%$ en el control) y una percepción positiva de la capacidad de manejo en consulta $(27,3 \%$ y $30 \%$ respectivamente), se encuentran también respuestas relativas a no tener suficiente tiempo para atender a estos pacientes (36,4\% y $30 \%$ en cada centro), percepción negativa de la capacidad de manejo en consulta $(18,2 \%$ y $10 \%)$ e incluso cierta sensación de incomodidad $(9,1 \%$ y $20 \%$ respectivamente).

3.3. Valoración del médico de atención primaria sobre la calidad de la información proveniente de salud mental

Los médicos del centro experimental valoran más positivamente $(\alpha<0,05)$ la calidad de la información que les llega desde salud mental sobre aquellos pacientes que son devueltos a consulta de atención primaria, así como sobre aquellos que continúan en tratamiento en salud mental (Tabla 3). En ambos centros se observa además que la calidad de la información de los pacientes que continúan en tratamiento en salud mental es peor valorada que la de aquellos que son devueltos a atención primaria. 
Tabla 3

Valoración del MAP sobre la calidad de la información proveniente de SM

\begin{tabular}{l|c|c|c|c}
\hline & \multicolumn{2}{|c|}{ Media (DT) $)^{\mathrm{a}}$} & \multirow{2}{*}{ sig. $^{\mathrm{b}}$} \\
\cline { 2 - 3 } & $\begin{array}{c}\mathrm{M}^{\mathrm{a}} \text { de Guzmán } \\
(\mathrm{n}=11)\end{array}$ & $\begin{array}{c}\text { Luis Vives } \\
(\mathrm{n}=10)\end{array}$ & $\mathrm{U}=12^{* *}$ & 0,002 \\
\hline $\begin{array}{l}\text { Sobre pacientes derivados a SM } \\
\text { y devueltos a AP }(0 \text { nula-5 muy buena) }\end{array}$ & $4,18(0,98)$ & $2,5(0,85)$ & $\mathrm{U}=17^{* *}$ & 0,006 \\
\hline $\begin{array}{l}\text { Sobre pacientes derivados a SM y } \\
\text { que continúan en tratamiento en SM } \\
(0 \text { nula-5 muy buena) }\end{array}$ & $3,36(1,43)$ & $1,50(0,71)$ & & \\
\hline
\end{tabular}

a Aunque el estadístico U de Mann-Whitney no se calcula sobre las medias muestrales, se exponen éstas en la tabla para facilitar la comprensión.

b Los contrastes son bilaterales.

** Estadísticamente significativo para $\alpha<0,01$

3.4. Valoración del médico de atención primaria sobre la coordinación atención primaria- salud mental

La cantidad de contactos y la disponibilidad del equipo de salud mental son mejor valorados en el centro experimental que en el control $(\alpha<0,05)$ (Tabla 4). Asimismo, los médicos del centro experimental han recibido un número significativamente mayor $(\alpha<0,05)$ de cursos de formación continuada en salud mental que en el control, aunque no se observan diferencias significativas entre centros al valorar la calidad de estos cursos.

Si bien en el centro control un porcentaje importante de médicos de atención primaria dice conocer a algún profesional de salud mental (70\%), esta proporción se reduce drásticamente cuando se les pregunta si se han coordinado directamente con alguno de ellos (20\%), frente al $100 \%$ de médicos del centro experimental que dicen haber llevado a cabo tal coordinación. También llamativa resulta la diferencia en la valoración general de la coordinación actual salud mental - atención primaria: en una escala sobre 10, el centro experimental otorga a la coordinación una puntuación de 8,36, frente al 2,89 del centro control. Estas diferencias resultan, una vez más, estadísticamente significativas (con un criterio $\alpha<0,05)$.

3.5. Percepción de necesidades formativas en salud mental

Finalmente, las necesidades formativas percibidas por los médicos de atención primaria de ambos centros (Tabla 5) tienen que ver fundamentalmente con formación en distintas modalidades de terapia y en tratamiento de los trastornos mentales más frecuentes. En cualquier caso, las pruebas $\chi 2$ realizadas sobre tablas de contingencia no reflejan diferencias significativas (con un criterio $\alpha<$ 0,05 ) en la distribución de las respuestas entre ambos centros en ninguna de las categorías, de manera que cualquier análisis que se pueda derivar de estos datos 
Tabla 4

Valoración del MAP sobre la coordinación AP-SM

\begin{tabular}{|c|c|c|c|c|}
\hline & \multicolumn{2}{|c|}{$\operatorname{Media}^{\text {a }}(\mathrm{DT}) / \mathrm{n}(\%)$} & \multirow[b]{2}{*}{$\mathrm{U} / \mathrm{x}^{2}$} & \multirow[b]{2}{*}{ sig. ${ }^{b}$} \\
\hline & $\begin{array}{l}\mathrm{M}^{\mathrm{a}} \text { de Guzmán } \\
(\mathrm{n}=11)\end{array}$ & $\begin{array}{l}\text { Luis Vives } \\
(\mathrm{n}=10)\end{array}$ & & \\
\hline $\begin{array}{l}\text { Cantidad de contactos con equipo de SM } \\
\text { (0 nulos-3 excesivos) }\end{array}$ & 1,82 & 0,7 & $\mathrm{U}=7 * *$ & $\mathrm{p}<0,001$ \\
\hline Disponibilidad del equipo de SM ( 0 nula- 3 excelente) & 2,73 & 1,11 & $\mathrm{U}=6^{* *}$ & $\mathrm{p}<0,001$ \\
\hline Utilidad de la coordinación ( 0 nula- 4 muy buena) & 3,55 & 2,7 & $\mathrm{U}=31,5$ & 0,099 \\
\hline $\begin{array}{l}\text { Número de cursos recibidos en el último año de } \\
\text { Formación Continuada de SM }\end{array}$ & 3,8 & 1,44 & $\mathrm{U}=10,5^{* *}$ & 0,003 \\
\hline $\begin{array}{l}\text { Calidad de cursos de Formación Continuada en SM } \\
(0 \text { muy malos- } 5 \text { excelentes })\end{array}$ & 4,09 & 3,60 & $\mathrm{U}=17,5$ & 0,267 \\
\hline Conocimiento de profesionales de SM (Sí/No) & $11(100 \%)$ & $7(70 \%)$ & $x^{2}=3,85$ & 0,09 \\
\hline Coordinación con profesionales de SM (Sí/No) & $11(100 \%)$ & $2(20 \%)$ & $\mathrm{x}^{2}=14,215^{* *}$ & $p<0,001$ \\
\hline Calidad de la coordinación actual SM-AP $(0-10)$ & 8,36 & 2,89 & $\mathrm{U}=0^{* *}$ & $\mathrm{p}<0,001$ \\
\hline $\begin{array}{l}\text { Aspectos de la coordinación con SM-AP } \\
\text { que serían más útiles } \\
\text { Colaboración, asesoría semanal, intercambio } \\
\text { de información }\end{array}$ & $6(54,5 \%)$ & $5(50 \%)$ & $\mathrm{x}^{2}=0,043$ & 0,59 \\
\hline Formación continuada & $5(45,5 \%)$ & $1(10 \%)$ & $x^{2}=3,226$ & 0,094 \\
\hline Mayor accesibilidad al profesional de SM & 0 & $3(30 \%)$ & $\mathrm{x}^{2}=3,85$ & 0,09 \\
\hline Otros & $4(36,4 \%)$ & $2(20 \%)$ & $x^{2}=0,687$ & 0,367 \\
\hline
\end{tabular}

a Aunque el estadístico U de Mann-Whitney no se calcula sobre las medias muestrales, se exponen éstas en la tabla para facilitar la comprensión.

b Los contrastes son bilaterales.

** Estadísticamente significativo para $\alpha<0,01$

debe quedarse en el terreno descriptivo, sin posibilidad de generalizar los resultados a la población.

3.6. Impacto de la experiencia en la tasa de derivaciones a salud mental

La Tabla 6 muestra la tasa de derivaciones a salud mental efectuadas por el centro de atención primaria $\mathrm{M}^{\mathrm{a}}$ de Guzmán (donde se realizó la experiencia) en comparación con la tasa de los demás centros de atención primaria del municipio de Alcalá de Henares (el resto de centros de atención primaria del Área se representan mediante un número). La notable diferencia entre el centro experimental y el resto de centros animan a pensar que la experiencia efectivamente sirve para reducir el número de derivaciones efectuadas a atención especializada. 
Tabla 5

Percepción de necesidades formativas en SM

\begin{tabular}{l|c|c|c|c}
\hline & \multicolumn{2}{|c|}{$\mathrm{n}(\%)$} & \multirow{2}{*}{$\mathrm{x}^{2}$} & \multirow{2}{*}{ sig. $^{\mathrm{a}}$} \\
\cline { 2 - 3 } & $\begin{array}{c}\mathrm{M}^{\mathrm{a}} \text { de Guzmán } \\
(\mathrm{n}=11)\end{array}$ & $\begin{array}{c}\text { Luis Vives } \\
(\mathrm{n}=10)\end{array}$ & & \\
\hline Temas en los que se perciben necesidades de & & & & \\
formación en SM: & $2(18,2 \%)$ & $3(30 \%)$ & 0,403 & 0,450 \\
$\quad$ Formación en distintas terapias & $4(36,4 \%)$ & 0 & 4,492 & 0,055 \\
Manejo cotidiano de personalidades difíciles & $2(18,2 \%)$ & $2(20 \%)$ & 0,011 & 0,669 \\
Tratamientos para ttnos. mentales más frecuentes & $2(18,2 \%)$ & $1(10 \%)$ & 0,286 & 0,538 \\
Respuesta afirmativa general & $5(45,5 \%)$ & $5(50 \%)$ & 0,043 & 0,59 \\
Otros & & & \\
\hline
\end{tabular}

a Todos los contrastes son bilaterales.

Tabla 6

Tasa de derivación a SM (número de derivaciones por mil habitantes)

Datos acumulados año 2008

\begin{tabular}{|c|c|}
\hline EAP 1.1 & 24,83 \\
\hline EAP 1.2 & 10,28 \\
\hline EAP 2 & 19,37 \\
\hline EAP 3.1 & 21,29 \\
\hline EAP 3.2 & 12,03 \\
\hline EAP 4 & 12,25 \\
\hline EAP 5 & 15,26 \\
\hline EAP 6 & 14,6 \\
\hline EAP 7 & 16,4 \\
\hline EAP 8 & 14,94 \\
\hline EAP 9.1 & 20,36 \\
\hline EAP 9.2 & 13,98 \\
\hline EAP Ma DE GUZMAN I & 3,25 \\
\hline EAP Ma DE GUZMAN II & 3,71 \\
\hline EAP 11 & 16,34 \\
\hline EAP 12 & 18,26 \\
\hline EAP 13 & 22,31 \\
\hline EAP 14 & 18,51 \\
\hline EAP 15 & 21,65 \\
\hline EAP 16 & 19,78 \\
\hline EAP 17 & 12,9 \\
\hline EAP 18 & 16,98 \\
\hline EAP 19 & 13,13 \\
\hline
\end{tabular}


ORIGINALES Y REVISIONES

\section{Discusión.}

Al comparar las respuestas de los médicos de atención primaria de cada uno de los centros se han obtenido algunas diferencias estadísticamente significativas (con, al menos, $\alpha<0,05$ ) en las valoraciones que se hacen sobre distintos aspectos de la relación atención primaria-salud mental: calidad de la información que aporta salud mental sobre los pacientes derivados a ese nivel (tanto aquellos devueltos a atención primaria, como aquellos que continúan seguimiento en salud mental); cantidad de contactos con el equipo de salud mental; disponibilidad del equipo de salud mental; cantidad de cursos de formación continuada específicos de salud mental; haberse coordinado alguna vez con un profesional de salud mental; y la valoración de la calidad de la coordinación actual. Todas estas diferencias apuntan en la misma dirección: los médicos de atención primaria del centro experimental se coordinan con más frecuencia con salud mental, encuentran mayor utilidad en esta coordinación y la consideran de mejor calidad.

En ambos centros estudiados, los factores que con más frecuencia determinan la derivación de un caso a salud mental son la falta de respuesta al tratamiento farmacológico y la solicitud del propio paciente de consultar con un especialista (Tabla 2). Esto da pie a preguntarse si al menos parte de esta demanda podría mantenerse en el nivel de atención primaria en caso de que el médico de atención primaria estuviera dotado de un conjunto básico de habilidades (de entrevista, de exploración de emociones, de análisis de la demanda) que le permitieran manejar en su consulta algunos de estos casos que no respondan al tratamiento farmacológico o que le pidan directamente tratamiento especializado en salud mental. La atención a pacientes de mayor gravedad o cronicidad puede suponer un estresor adicional para el médico, por lo que el trabajo dirigido a ayudarle con esta carga puede resultar provechoso. Es de esperar que un entrenamiento adecuado a los médicos de atención primaria se refleje en el tipo y cantidad de derivaciones que hagan a salud mental.

Los médicos de atención primaria dicen emplear con cierta frecuencia alguna forma de apoyo psicológico no estructurado, pero sería importante conocer a qué se refieren con esto, qué es lo que hacen exactamente, y si hay espacio en ello para la mejora. Asimismo, queda abierto el debate sobre la conveniencia o no de entrenar a médicos de atención primaria en algún abordaje psicológico estructurado.

Llama la atención la mayor demanda de ayuda a los profesionales de salud mental que hacen los médicos de atención primaria del centro experimental, en comparación con el control, para manejar estos pacientes en su consulta (Tabla 2). Parecería que "a más recursos invertidos, más piden". Una explicación alternativa podría ser que el contacto directo con los especialistas de salud mental les 
hubiera hecho más conscientes de aspectos del trabajo con pacientes (de salud mental u otros) que podrían beneficiarse de un entrenamiento por parte de los profesionales de atención especializada. De esta manera, la presencia de los profesionales de salud mental en el centro de salud no habría generado una nueva necesidad, sino que habría permitido detectar una necesidad que ya existía.

No se han encontrado diferencias estadísticamente significativas entre centros en ninguna de las categorías de respuesta de las preguntas abiertas. Para este trabajo fue un único juez no ciego el que clasificó las respuestas en categorías semánticas; es posible que el uso de dos jueces ciegos y la búsqueda de acuerdo interjueces hubiera dado lugar a otro resultado. Asimismo es necesario plantearse si acaso las preguntas abiertas estaban formuladas en términos demasiado generales como para producir respuestas suficientemente concretas que permitieran detectar diferencias entre los dos centros.

No obstante, los médicos de atención primaria del centro donde se estaba realizando la experiencia piloto de coordinación se mostraban significativamente más satisfechos con los contactos con salud mental que el otro centro. Dentro de que éste no dejaría de ser un resultado esperable, hace pensar que esta satisfacción debería deberse a algo más que a la mera presencia de los profesionales de salud mental. Futuros trabajos deberían dirigirse a detectar de qué formas concretas se estarían beneficiando de la coordinación los médicos de atención primaria del centro experimental. El empleo de entrevistas en profundidad con algunos de ellos podría aclarar en parte este punto.

Pero más allá de estos resultados, especialmente llamativa resulta la reducción de derivaciones a salud mental en el centro objeto de estudio en comparación con otros centros de atención primaria. Este trabajo muestra cómo es posible un modelo alternativo de coordinación entre atención primaria y salud mental con un importante potencial para reducir el número de derivaciones. Este nuevo sistema, con el que los médicos de atención primaria se muestran altamente satisfechos, sienta los cimientos de una nueva forma de trabajo en equipo, en el que los médicos de atención primaria no perciban a los profesionales de salud mental de una manera tan lejana, y éstos sean más sensibles a las realidades y necesidades de los médicos de atención primaria en su trabajo cotidiano. 
Anexo: Cuestionario de evaluación utilizado

\section{CUESTIONARIO PARA MÉDICOS DE AP SOBRE SALUD MENTAL}

Centro de salud en el que trabajas:

Edad: Sexo: Años trabajando como MAP en el sector público:

Situación laboral (fijo / interino / otras): Turno de trabajo:

1. ¿Qué tipo de problemas psicológicos/psiquiátricos acuden a tu consulta con mayor frecuencia?

2. De estos pacientes, ¿cuáles decides derivar a SM con más frecuencia? ¿Hay algún factor que condicione o favorezca la derivación de forma más rápida o evidente?

3. De los pacientes con problemas psicológicos/ psiquiátricos que atiendes y no derivas al nivel especializado (o te son inmediatamente devueltos desde SM por padecer problemas atendibles desde AP), ¿cómo valoras la atención en salud mental que prestas desde AP?

(Desde 0 muy mala, 1 mala, 2 deficiente, 3 adecuada, 4 satisfactoria, 5 muy satisfactoria)

a. ¿Qué sueles hacer con estos pacientes? (Nunca, rara vez, a veces, frecuentemente, siempre)

i. Dar fármacos

ii. Apoyo psicológico no estructurado

iii. Algún tipo de abordaje psicológico estructurado. ¿Cuál?

b. ¿Cómo consideras que podría ser la ayuda que necesitas desde el equipo de Salud Mental con estos pacientes con problemas psicológicos que atiendes en tu consulta?

4. ¿Cómo describirías tu percepción del paciente con problemas psicológicos/ psiquiátricos y tu reacción frente al mismo cuando llega a tu consulta?

5. ¿Qué te parece la información disponible sobre los pacientes evaluados en SM y derivados nuevamente a AP? ( Nula, mala, escasa, adecuada, buena, muy buena)

6. ¿Qué te parece la información disponible sobre los pacientes en tratamiento en SM?

(Nula, mala, escasa, adecuada, buena, muy buena)

7. Valoración de la coordinación con el equipo de Salud mental

a. Cantidad de contactos con el equipo de SM (teléfono, presencial, sesiones...)

(Nulos, escasos, adecuados, excesivos)

b. Disponibilidad del equipo de SM (Nula, escasa, adecuada, excelente)

c. Utilidad de la coordinación para la consulta de AP (Nula, escasa, adecuada, buena, muy buena)

d. Cantidad de cursos de formación continuada en Salud Mental (de 0 a >5 al año)

e. Calidad de dichos cursos (de 0: muy malos a 5: excelentes)

f. ¿Conoces a alguno de los psiquiatras/ psicólogos del equipo de SM? ¿Te has coordinado con alguno?

g. Valoración general de la coordinación con Salud Mental en la actualidad (de 0 muy mala a 10 óptima)

h. ¿Qué aspectos de la coordinación con AP valorarías más o te serían más útiles?

8. ¿Percibes necesidades de formación en Salud Mental? ¿en algún tema especial? 
ORIGINALES Y REVISIONES

\section{BIBLIOGRAFÍA:}

(1) Desviat, M., La reforma psiquiátrica, Madrid, DOR, 1993.

(2) Comisión Ministerial para la Reforma Psiquiátrica, Informe de la Comisión Ministerial para la Reforma Psiquiátrica, Madrid, Ministerio de Sanidad y Consumo, 1985.

(3) Ley 25-4-1986 Ley General de Sanidad BOE 29-4-1986 Núm.102, Ley 25-4-1986 Ley General de Sanidad BOE 29-4-1986 Núm.102, (1986).

(4) Fernández Liria, A.; Diéguez Porres, M., "La reforma psiquiátrica en España: aspectos diferenciales”, Revista de la Administración Sanitaria, 1998, II, 5, pp. 85-102.

(5) Fernández Liria, A., "La reforma psiquiátrica en España: En qué (y en qué no) metimos la pata”, Psiquiatría Pública, 1997, IX, 3, 167-175.

(6) Ministerio de Sanidad y Consumo, Estrategia en Salud Mental, Madrid, Ministerio de Sanidad y Consumo, 2007.

(7) Tızón, J. L., "Posibilidades, medios y sistemas de relación entre los equipos de salud mental y los equipos de atención primaria", en Otero, J. F. (ed.), Trastornos psiquiátricos y atención primaria, Madrid, Doyma, 1995, pp. 31-52.

(8) Martínez Azurmendi, O., "Coordinación con atención primaria: una visión posibilista", Norte de Salud Mental, 2001, IV, 13, pp. 83-91.

(9) Bower P, Sibbald B. Profesionales sanitarios de Salud Mental dentro de los servicios de Atención Primaria: efectos sobre su práctica profesional. En: La Biblioteca Cochrane Plus, 2007, 4. Oxford.

(10) Strathdee G, Williams P. A survey of psychiatrists in primary care: the silent growth of a new service. J R Coll Gen Pract. 1984;34:615-18.

(11) Gualtero R, Turek L. Salud mental: encuesta a los MAP. Rev. Asoc. Esp. Neuropsiquiatría. 1999;70:225-34.

(12) Brazeau, C., Y otros, "Collaboration between mental health professionals and family physicians: a survey of New Jersey Family Physicians", Primary Care Companion to the Journal of Clinical Psychiatry, 2005, VII, 1, pp. 12-14.

* Carlos Hornillos Jerez (1). Eva Rodríguez Pedraza (1). Francisco González Aguado (1). Alberto Fernández Liria (1) (2). (1) Servicios de Salud Mental del Área 3 de Madrid, Hospital Príncipe de Asturias. (2) Universidad de Alcalá

Correspondencia: Eva Rodríguez Pedraza. Secretaría de Psiquiatría. Planta $4^{\circ}$ D. Hospital Universitario Príncipe de Asturias. Ctra. Alcalá-Meco, s/n. 28805 Alcalá de Henares (Madrid). Teléfono: 918878100 Ext. 2523. Correo electrónico: evapedraza@yahoo.es

** Recibido: 8/11/2008. 